\begin{tabular}{|c|c|}
\hline Title & Magnetic Sensor Devices Based on Ordered Planar A rrangements of MnA s Nanocluster \\
\hline Author(s) & Heiliger, Christian; Czerner, Michael; Klar, Peter J.; Hara, Shinjiroh \\
\hline Citation & $\begin{array}{l}\text { IEEE Transactions on Magnetics, 46(6), 1702-1704 } \\
\text { https://doi.org/10.1109/ MA G.2010.2041194 }\end{array}$ \\
\hline Issue Date & $2010-06$ \\
\hline Doc URL & http:/hdl.handle.net/2115/43113 \\
\hline Rights & $\begin{array}{l}\text { C 2010 IEEE. Personal use of this material is permitted. However, permission to reprint/republish this material for } \\
\text { advertising or promotional purposes or for creating new collective works for resale or redistribution to servers or lists, } \\
\text { or to reuse any copyrighted component of this work in other works must be obtained from the IEEE. }\end{array}$ \\
\hline Type & article \\
\hline File Information & TM46-6_1702-1704.pdf \\
\hline
\end{tabular}

Instructions for use 


\title{
Magnetic Sensor Devices Based on Ordered Planar Arrangements of MnAs Nanocluster
}

\author{
Christian Heiliger ${ }^{1}$, Michael Czerner ${ }^{1}$, Peter J. Klarr ${ }^{1}$, and Shinjiroh Hara ${ }^{2}$ \\ ${ }^{1}$ I. Physikalisches Institut, Justus Liebig University, Giessen, 35392, Germany \\ ${ }^{2}$ Research Center for Integrated Quantum Electronics, Hokkaido University, Sapporo 060-8628, Japan
}

\begin{abstract}
We propose planar magneto-electronic devices based on accurately positioned and shaped ferromagnetic MnAs nanoclusters in NiAs structure. The prototype discussed consists of a small hexagon-shaped cluster sandwiched between two elongated clusters with fixed magnetic orientations. The magnetization of the center cluster is free and can be rotated by an external magnetic field. Our ab initio calculations yield a strong dependence of the conductance as a function of the direction of the external magnetic field which exhibits an in-plane symmetry of 360 degrees.
\end{abstract}

Index Terms-Magnetic device, magnetic domains, magnetoresistance, nanotechnology.

\section{INTRODUCTION}

$\mathbf{T}$ HE DISCOVERY of the giant magneto-resistance (GMR) effect [1] opened the field to spintronics where the electron's spin is exploited in addition to its charge. Important applications of GMR and the related tunneling magneto-resistance (TMR) [2] devices are read heads for hard disks, storage elements in magnetic memories, and magnetic sensors.

So far, the technological application of MnAs clusters has been mainly restricted to magnetic devices containing layers with randomly arranged MnAs nanoclusters, i.e., where the mean distance between clusters is much smaller than the characteristic device size and the properties of the ensemble of MnAs clusters is used to contribute to the function of the device [3]-[5]. The recently introduced selective-area metal-organic vapor-phase epitaxy (SAMOVPE) process offers novel ways of controlling size, shape, and position of MnAs clusters on the submicron scale [6], [7] and thus also to tune the magnetic properties of individual MnAs clusters. In particular, the clusters are grown with the $\mathrm{c}$ axis perpendicular to the substrate. Bulk hexagonal MnAs exhibit a hard magnetic axis along $\mathrm{c}$ and an easy plane of magnetization perpendicular to it. Therefore, the behavior of the in-plane magnetization is strongly affected by the shape of the clusters. This forms a sound basis for using such clusters as building blocks for novel devices on the nanoscale. It is envisioned, that controlling the structure of individual clusters (e.g., size, shape, orientation) and the coupling between them (e.g., position), in conjunction with theoretical modelling on a predictive level, can be used to design a new class of planar magneto-electronic devices. The planar device geometry differs from typical GMR and TMR stack-like vertical arrangements. This may turn out to be advantageous for some applications.

Similar to the giant magneto-resistance effect or the tunneling magneto-resistance effect, the conductance through a device based on MnAs clusters as building blocks depends on the relative magnetic orientation of the ferromagnetic clusters

Manuscript received October 30, 2009; accepted January 09, 2010. Current version published May 19, 2010. Corresponding author: C. Heiliger (e-mail: Christian.heiliger@physik.uni-giessen.de).

Color versions of one or more of the figures in this paper are available online at http://ieeexplore.ieee.org.

Digital Object Identifier 10.1109/TMAG.2010.2041194 with respect to each other. It is quantified by the magneto-resistance ratio $\left(g_{P}-g_{A P}\right) / g_{A P}$ where $g_{P}\left(g_{A P}\right)$ is the conductance for the parallel (antiparallel) alignment of the ferromagnetic layers. For typical devices the GMR ratio is on the order of $30 \%$ whereas for modern TMR devices with $\mathrm{MgO}$ as barrier material TMR ratios of several $100 \%$ are possible [8].

Theoretical investigations of MnAs predict that in the zincblende crystal structure the system is half metallic [9] which is an ideal starting point for spintronic devices. However, the MnAs clusters obtained by SAMOVPE are of hexagonal NiAs crystal structure. Theoretical investigations of this structure reveal a metallic system with a relatively low spin polarization at the Fermi energy [10], [11].

\section{METHOD}

We use an ab initio Korringa Kohn Rostoker (KKR) Green's function method based on density functional theory within the local spin-density approximation to calculate the band structure of hexagonal MnAs. The transport calculations are based on the Keldysh non-equilibrium Green's function formalism implemented within the KKR method [12]. Thereby, a treatment of the magnetic moments with a noncollinear magnetic order is possible.

The lattice constants are chosen to be $\mathrm{a}=3.725 \AA$ and $\mathrm{c}=5.713 \AA$ from [11]. The wave functions and Green's functions in the present calculations were expanded up to angular momenta $\mathrm{l}=3$, while the expansion of the charge density was taken into account up to $l=6$. We assumed spherical potentials in the atomic sphere approximation. Exchange and correlation effects were described using LDA. The resulting bulk magnetic moment per molecule is $2.7 \mu_{\mathrm{B}}$ in our calculation. For the transport calculation a frozen potential approximation is used. This means that for the assumed ideal interface between two MnAs clusters with different magnetic orientations the bulk potentials are used. In particular, an abrupt domain wall is assumed. Therefore, the calculation can be seen as an upper limit of what may be achieved. In reality, the domain wall is continuous leading to a reduction of the effects. It was shown that for domain walls in bulk ferromagnets the reduction is strong with increasing domain wall width [13]. However, the geometry of the device we discuss later contains a constriction. In such a geometry it was shown by Bruno [14] that the domain wall width can be much smaller compared to a bulk domain wall. 


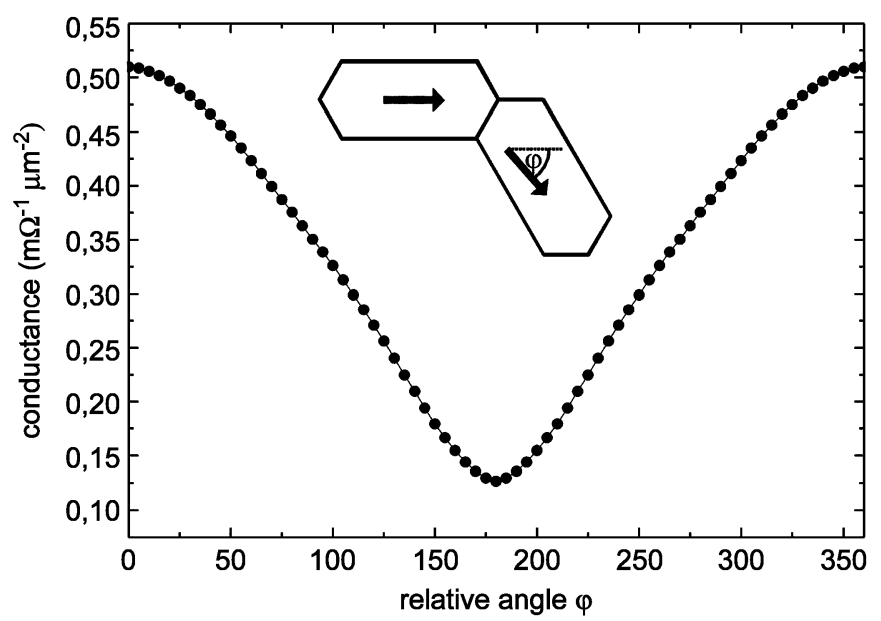

Fig. 1. Conductance values in units of the conductance quantum $g_{0}$ plotted in the 2-D Brillouin zone perpendicular to the transport direction for parallel (left) and antiparallel (right) layer magnetizations and different spin channels (up up (majority), down down (minority), down up (equivalent to up down)).

\section{RESULTS}

We start with a device consisting of two elongated MnAs clusters connecting at an m-plane at an angle of $120^{\circ}$ shown as the inset of Fig. 1. For the calculations this geometry is transferred to a structure with two semi-infinite MnAs leads with the interface parallel to the m-plane. Fig. 1 shows the conductance as a function of the relative angle between the two magnetizations of the MnAs clusters. We do not consider anisotropic effects in our theory. Therefore, only the relative angle is important. The transmission follows basically a cosine function. The magneto-resistance ratio in that case is very large of about $300 \%$. This is a surprising result because the spin polarization in the parallel configuration is small with only $40 \%$. The origin of the high magneto-resistance ratio is that the contributing states are at different areas within the Brillouin zone for the two spin channels. Assuming coherent transport across the interface the wave vector parallel to the interface is conserved. Consequently, for the antiparallel configuration there is a mismatch of the states in the different spin channels leading to a strong reduction of the conductance in the antiparallel configuration. Fig. 2 shows the conductance within the 2-D Brillouin zone perpendicular to the transport direction for the different spin channels [up up (majority), down down (minority), up down (equivalent to down up)]. In these maps, one can analyze the transport properties of the individual in-plane wave vectors. In the majority spin channel, almost all states contribute to the transport, but states at the corners of the Brillouin zone have a particularly large transmission probability. In contrast, there are fewer states in the minority spin channel contributing to the transport. In particular, at areas with a large transmission probability of the majority states there is only a small or even vanishing transmission probability for the minority states. Consequently, the transmission probabilities for the states in the mixed spin channels in the antiparallel configuration are small, which leads to the very large magneto-resistance ratio. This effect of different contributing areas within the 2-D Brillouin zone is well known for other systems like tunnel junctions where this effect is also important to obtain large TMR ratios [15]. This demonstrates the huge potential of MnAs for spintronic applications.

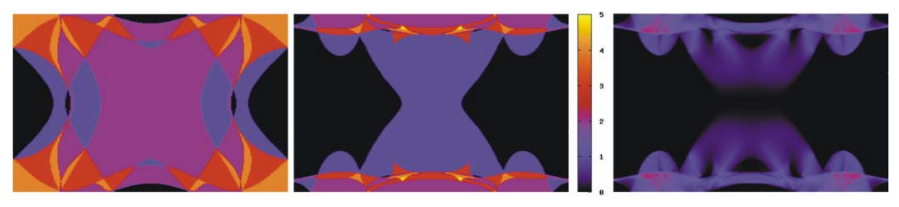

Fig. 2. Conductance of a two-cluster device (inset) as a function of the orientation of the magnetizations.
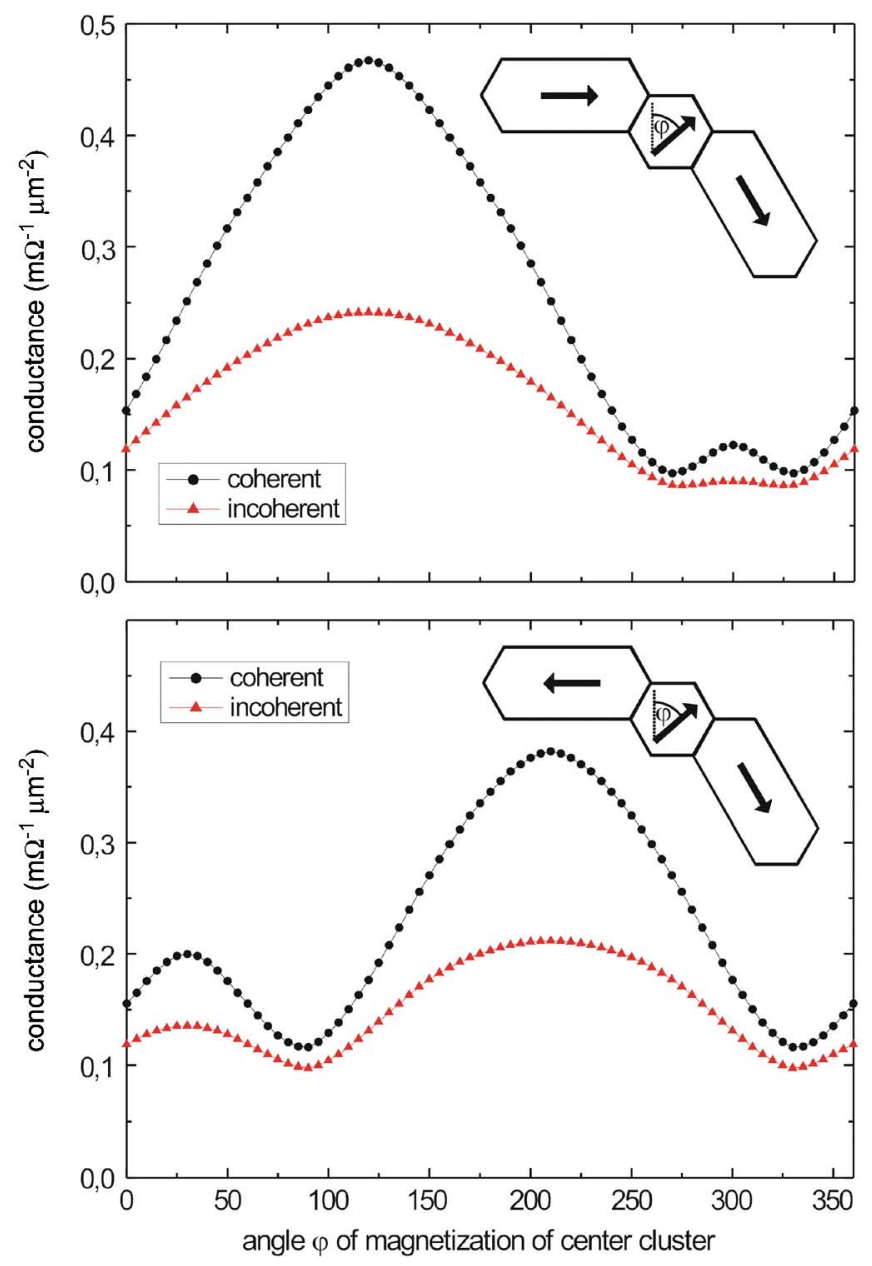

Fig. 3. Conductance of a three-cluster device as a function of the orientation of the magnetization of the center cluster for fixed magnetization orientations of the elongated clusters. The orientations of the magnetizations of the elongated clusters are given in the insets.

We continue our discussion with a proposal of a three-cluster device that can be used as a magnetic sensor. For this purpose, we sandwich a small hexagon-shaped cluster between two elongated MnAs clusters. The reason for this geometry is that due to shape anisotropy the orientation in the elongated clusters is fixed whereas for the small cluster the orientation of the magnetization can be changed easily [6]. An important question is whether the transport across the two interfaces is coherent or incoherent. We assume that the transport across one interface is coherent but that the phase information may be lost reaching the second interface. Therefore, we performed coherent and incoherent calculations shown in Fig. 3. The latter is done by using the results of one interface from Fig. 1 and by assuming conductance in series for the two interfaces. In the coherent calculation the transport through the whole junction is coherent leading 
to a stronger dependence of the conductance versus the angle $\varphi$ in comparison to the incoherent treatment. Nevertheless, the change of conductance is still very large for the incoherent case. For the coherent case, the distance between the interfaces may be important. We performed a calculation for a doubled distance between interfaces but found no significant differences. An important result is that the conductance is a function of the full $360^{\circ}$ with a large change of the conductance. Therefore, the proposed device can be used as a very sensitive magnetic field sensor. The largest conductance is obtained when the magnetization of the center cluster lies between the orientations of the two elongated clusters. The comparison of top and bottom graph of Fig. 3 shows that additional functionality can be achieved by using the possibility to switch the magnetization orientation of one of the elongated clusters. The dependence of the conductance is then basically shifted by $90^{\circ}$. In addition, the relative height of both peaks is changing. The magnetic properties, e.g., the switching behavior, of the clusters and thus of the cluster arrangement can also be tuned by controlling cluster sizes, shapes, and positions. Therefore, additional possibilities for optimizing the magneto-resistance behavior in an applied magnetic field arise which make such cluster arrangements interesting for spin (or magneto-logic) circuits, memory devices, sensors, etc.

\section{CONCLUSIONS}

We demonstrate that planar ordered arrangements of coupled ferromagnetic MnAs clusters possess a huge potential for novel magneto-electronic devices on the submicron scale. Although the spin polarization at the Fermi level of hexagonal MnAs itself is low the magneto-resistance ratio exceeds $300 \%$. As proof of principle, we propose a magnetic sensor device which is very sensitive to the direction of the magnetic field yielding a $360^{\circ}$ full turn dependence of its conductance. For applications the domain wall has to be as sharp as possible. Another approach could be to stop the growth process just before the clusters are connected and deposit a non-magnetic material to construct GMR structures. That way, planar magneto electronic devices with a large magneto resistance effect are at hand.

\section{REFERENCES}

[1] G. Binasch, P. Grünberg, F. Saurenbach, and W. Zinn, Phys. Rev. B, vol. 39, p. 4828, 1989

[2] J. Moodera, L. Kinder, T. Wong, and R. Meservey, Phys. Rev. Lett., vol. 74, p. 3273, 1995.

[3] F. L. Bloom, A. C. Young, R. C. Myers, E. R. Brown, A. C. Gossard, and E. G. Gwinn, J. Vac. Sci. Technol. B, vol. 24, p. 1639, 2006.

[4] P. N. Hai, S. Ohya, M. Tanaka, S. E. Barnes, and S. Maekawa, Nature, vol. 458, p. 489, 2009

[5] M. Shimizu, M. Miyamura, and M. Tanaka, Appl. Phys. Lett., vol. 78, p. 1523,2001

[6] S. Ito, S. Hara, T. Wakatsuki, and T. Fukui, Appl. Phys. Lett., vol. 94, p. 243117, 2009.

[7] T. Wakatsuki, S. Hara, S. Ito, D. Kawamura, and T. Fukui, Jpn J. Appl. Phys., vol. 48, p. 04C137, 2009.

[8] C. Heiliger, P. Zahn, and I. Mertig, Mater. Today, vol. 9, p. 46, 2006.

[9] S. Sanvito and N. A. Hill, Phys. Rev. B, vol. 62, p. 15553, 2000.

[10] A. Continenza, S. Picozzi, W. T. Geng, and A. J. Freeman, Phys. Rev. $B$, vol. 64, p. 085204, 2001.

[11] M. Shirai and Y. Tokioka, J. Electron Spectrosc. Relat. Phenom., vol. 88-91, p. 357, 1998.

[12] C. Heiliger, M. Czerner, B. Y. Yavorsky, I. Mertig, and M. D. Stiles, J. Appl. Phys., vol. 103, p. 07A709, 2008.

[13] J. B. A. N. van Hoof, K. M. Schep, A. Brataas, G. E. W. Bauer, and P. J. Kelly, Phys. Rev. B, vol. 59, p. 138, 1999.

[14] P. Bruno, Phys. Rev. Lett., vol. 83, p. 2425, 1999.

[15] C. Heiliger, M. Gradhand, P. Zahn, and I. Mertig, Phys. Rev. Lett., vol. 99, p. 066804, 2007. 